\title{
Obsessive-Compulsive Symptoms and Related Sex Differences in Brain Structure: An MRI Study in Dutch Twins
}

\author{
Anouk den Braber, Eco J.C. de Geus, Dorret I. Boomsma, and Dennis van 't Ent \\ Department of Biological Psychology, VU University, van der Boechorststraat 1, 1081 BT, Amsterdam, The Netherlands
}

\begin{abstract}
Neuroimaging studies have indicated abnormalities in cortico-striato-thalamo-cortical circuits in obsessivecompulsive disorder patients, but results have not been consistent. Since there are significant sex differences in human brain anatomy and obsessive-compulsive symptomatology and its developmental trajectories tend to be distinct in males and females, we investigated whether sex is a potential source of heterogeneity in neuroimaging studies on obsessive-compulsive symptoms. We selected male and female twin pairs who were concordant for scoring either high or low for obsessive-compulsive symptoms and a group of discordant pairs where one twin scored high and the co-twin scored low. The design included 24 opposite-sex twin pairs. Magnetic resonance imaging scans of 31 males scoring high for obsessive-compulsive symptoms, 41 low-scoring males, 58 high-scoring females, and 73 low-scoring females were analyzed and the interaction of obsessive-compulsive symptoms by sex on gray matter volume was assessed using voxel-based morphometry. An obsessive-compulsive symptom by sex interaction was observed for the left middle temporal gyrus, the right middle temporal gyrus, and the right precuneus. These interactions acted to reduce or hide a main effect in our study and illustrate the importance of taking sex into account when investigating the neurobiology of obsessive-compulsive symptoms.
\end{abstract}

Keywords: structural magnetic resonance imaging, obsessive-compulsive symptom by sex interaction, gray matter volume, precuneus, middle temporal cortex

Obsessive-compulsive (OC) symptoms are characterized by recurrent, persistent, and intrusive anxiety-provoking thoughts/images (obsessions) and subsequent repetitive behaviors (compulsions) performed to reduce anxiety and/or distress caused by obsessions. When a person has obsessions and/or performs compulsions for more than an hour a day and these thoughts and rituals significantly interfere with daily life routines, the person fulfills the criteria for obsessive-compulsive disorder (OCD). The lifetime prevalence of OCD is 0.5-2\% (American Psychiatric Association, 1994; Grabe et al., 2000), but obsessions are much more prevalent in the general population - as high as $72 \%$ (Rachman \& de Silva, 1978; Salkovskis \& Harrison, 1984) and the prevalence of OC symptoms reaches up to $20 \%$ (Fullana et al., 2009).

Neuroimaging studies have indicated neurobiological changes underlying the psychological and behavioral dysfunction of OCD. Results from structural and functional magnetic resonance imaging (MRI) studies mainly point to volume differences and altered regional brain activation in the ventral prefrontal cortex, dorsolateral prefrontal cortex, basal ganglia, anterior cingulate cortex (ACC), and thalamus (Menzies et al., 2008; Radua \& Mataix-Cols, 2009; Radua et al., 2010; Rotge et al., 2009). These findings contributed to the widely accepted neuroanatomical model of OCD involving the direct and indirect corticostriato-thalamo-cortical (CSTC) loops (Mataix-Cols \& van den Heuvel, 2006; Saxena \& Rauch, 2000). It has been hypothesized that an imbalance between these loops, resulting in a hyperactive ventral and hypoactive dorsal

RECEIVED 1 February 2013; ACCEPTED 7 February 2013.

ADDRESS FOR CORRESPONDENCE: Anouk den Braber, Department of Biological Psychology, VU University, van der Boechorststraat 1, $1081 \mathrm{BT}$, Amsterdam, The Netherlands. E-mail: a.den.braber@vu.nl 
frontal-striatal system, might mediate OC symptomatology (Mataix-Cols \& van den Heuvel, 2006; Saxena \& Rauch, 2000).

In spite of the convergence on the same brain regions, inconsistencies in the direction of OCD effects have been reported for volumetric differences of the implicated brain areas (larger vs. smaller) as well as their metabolism (hypo- or hyperactivation; Friedlander \& Desrocher, 2006; Menzies et al., 2008). These inconsistencies might be due to methodological differences between studies (e.g., differences in sample size, scanning modalities/parameters, and analysis methods), but they may also reflect heterogeneity in the patient groups scanned. For instance, we have shown that brain regions were affected differently in subjects characterized by high environmental risk for OC symptoms than in those characterized by high genetic risk for OC symptoms (den Braber et al., 2010, 2011). Here we hypothesize that sex is a second potential source of heterogeneity. The brains of males and females begin to differ in an early developmental stage through the action of sex-specific factors such as hormonal, genetic, and epigenetic factors (McCarthy \& Arnold, 2011), and sex-specific maturation continues during puberty and adolescence (Sisk \& Zehr, 2005). Postmortem and in vivo imaging studies of both children and adults consistently reported that males have approximately 9-12\% larger brain volumes than females. Apart from global volume differences, regional sexual dimorphisms have also been reported, primarily for areas with high numbers of sex steroid receptors. After correcting for total brain volume, males tend to have larger gray matter volumes in the amygdala and hypothalamus, whereas females tend to have larger orbitofrontal, hippocampal, and caudate volumes (for review, see Cosgrove et al., 2007; Lenroot \& Giedd, 2010). Sex-specific differences in the healthy brain also highlight the need to evaluate sex differences in the association of brain structure with neuropsychiatric disorders, especially those that differ in prevalence and/or symptoms between males and females such as OCD (Bogetto et al., 1999; Castle et al., 1995; Labad et al., 2008; Lensi et al., 1996; Noshirvani et al., 1991; Tukel et al., 2004).

The present study examines differences between males and females with low or high OC symptomatology, focusing specifically on gray matter volumes. Our study design included twin pairs who were discordant for OC symptoms or concordant for either high or low OC symptoms, including a group of 24 opposite-sex twin pairs. We hypothesize to find sex moderation of OC symptomatology effects in brain areas that were already implicated in OC symptoms in mixed male-female samples and have high levels of sex steroid receptors, that is, the striatum, thalamus, insula, ACC, and frontal, temporolimbic, and parietal areas. We further explore the existence of 'crossed line interactions' where an opposite effect of OC symptomatology in males and females may have acted to hide a main effect in mixed sex samples.

\section{Methods and Materials \\ Participants}

Participants were recruited from an ongoing study in the Netherlands Twin Register (NTR) that investigates environmental and genetic influences on OC symptoms (den Braber et al., 2010). Surveys were sent to twin families, including the Padua Inventory Abbreviated (PI-R-ABBR; Cath et al., 2008; van Oppen et al., 1995). Completed PIR-ABBR questionnaires were returned by 20,204 subjects (including 9,512 twins and 2,403 siblings). From this sample we selected twin and sibling pairs in the age range of 18 to 60 years who scored very high ( 5 opposite-sex twin pairs, 13 siblings, and 23 monozygotic twin pairs), very low (19 opposite-sex and 28 monozygotic twin pairs), or very discordant (20 monozygotic twin pairs) for OC symptoms. A subject was classified as high scoring for OC symptoms if the PI-R-ABBR score was $\geq 15$. A subject was classified as low scoring for OC symptoms if the PI-R-ABBR score was $\leq 7$. These PI-R-ABBR cutoff scores were derived from sensitivity and specificity measurements in an independent sample of OCD patients when compared with clinical controls ( $n=120$; mean scores $20.7, S D 8.1$, sensitivity 0.74 , and specificity 0.72 at the best cutoff point of 16; Cath et al., 2008). Exclusion criteria were brain damage, neurological disease, and contraindications for MRI (e.g., pregnancy, ferromagnetic fragments, clips and devices in the body, and claustrophobia). A final number of 203 subjects participated in our MRI study, including 58 high-scoring females, 31 high-scoring males, 73 low-scoring females and 41 lowscoring males (Table 1).

\section{Protocol}

Participants were administered diagnostic interviews and questionnaires, including questions on demography, lifeevents, an adapted form of the Yale-Brown ObsessiveCompulsive Scale (Goodman et al., 1989a, 1989b) to measure both lifetime and current OC symptoms, and severity, the State Trait Anxiety Inventory and State Trait Anger Scale (Spielberger et al., 1970, 1983), and the Mini International Neuropsychiatric Interview (MINI; Sheehan et al., 1998) to test for possible comorbidities. Comorbidities tested by the MINI include depression, panic disorder, agoraphobia, social phobia, post-traumatic stress disorder, and generalized anxiety disorder. Depressive symptoms were furthermore measured using the 13-item Beck Depression Inventory Short Form (BDI-R; Beck et al., 1961, 1974). In addition, participants were screened for the eight most common tics (head shaking, eye blinking, other facial tics, shoulder raising, expressing swear words/foul language/dirty words, sound making, growling, and throat clearing/coughing/sniffing). The ethical review board of the VU University Medical Centre approved the study. All participants provided written informed consent. 
TABLE 1

Sample Characteristics

\begin{tabular}{|c|c|c|c|c|c|c|c|}
\hline & & \multicolumn{2}{|c|}{ Males } & \multicolumn{2}{|c|}{ Females } & \multirow[b]{2}{*}{ OCS main effect } & \multirow[b]{2}{*}{$\begin{array}{l}p \\
\text { OCS } \times \text { sex } \\
\end{array}$} \\
\hline & & $\begin{array}{l}\text { High } \\
(n=31)\end{array}$ & $\begin{array}{l}\text { Low } \\
(n=41)\end{array}$ & $\begin{array}{l}\text { High } \\
(n=58)\end{array}$ & $\begin{array}{l}\text { Low } \\
(n=73)\end{array}$ & & \\
\hline \multirow[t]{4}{*}{ Sample characteristics } & Age (years; $S D$ ) & $30.52(8.19)$ & $31.32(8.68)$ & $35.90(10.42)$ & 36.55 (9.62) & .665 & .984 \\
\hline & Educational attainment (\% low/middle/high) & 12.9/38.7/48.4 & 7.3/26.8/65.9 & $17.2 / 36.2 / 46.6$ & $8.2 / 28.2 / 63.0$ & $.039^{\#}$ & .337 \\
\hline & Total intracranial volume (cc; SD) & $1564.32(122.71)$ & $1524.21(125.76)$ & $1358.78(109.99)$ & $1332.04(105.90)$ & .064 & $.003 *$ \\
\hline & & Mean $(S D)$ & Mean (SD) & Mean (SD) & Mean (SD) & & \\
\hline \multirow{8}{*}{$\begin{array}{l}\text { Obsessive-compulsive } \\
\text { symptoms }\end{array}$} & PI-R-ABBR (0-48) & $19.90(3.58)$ & $4.00(1.85)$ & $20.62(4.98)$ & $4.38(2.15)$ & $<.001 *$ & .733 \\
\hline & $\begin{array}{l}\text { Y-BOCS severity lifetime (0-40) } \\
\text { Y-BOCS symptoms }\end{array}$ & $8.65(6.87)$ & $3.24(4.85)$ & $10.33(7.22)$ & $4.19(5.88)$ & $<.001 *$ & .979 \\
\hline & Agressive/checking (0-14) & $2.52(2.11)$ & $0.88(1.05)$ & $3.17(2.50)$ & $0.96(1.17)$ & $<.001 *$ & .256 \\
\hline & Hoarding/saving (0-2) & $0.61(0.67)$ & $0.20(0.51)$ & $0.60(0.70)$ & $0.22(0.42)$ & $<.001 *$ & .842 \\
\hline & Symmetry/ordering (0-5) & $0.81(1.10)$ & $0.24(0.54)$ & $1.03(1.40)$ & $0.32(0.72)$ & $<.001 *$ & .362 \\
\hline & Washing/cleaning (0-12) & $1.23(1.65)$ & $0.46(0.90)$ & $2.12(2.32)$ & $0.56(1.11)$ & $<.001 *$ & .065 \\
\hline & Sexual/religious (0-8) & $1.42(1.63)$ & $0.49(0.75)$ & $1.88(1.86)$ & $0.47(0.73)$ & $<.001 *$ & 168 \\
\hline & Somatic $(0-2)$ & $0.55(0.72)$ & $0.49(0.22)$ & $0.72(0.77)$ & $0.29(0.54)$ & $<.001 *$ & .698 \\
\hline \multirow[t]{11}{*}{ Comorbidity } & MINI: & & & & & & \\
\hline & Depression (n) & 1 & 0 & 1 & 0 & 191 & .341 \\
\hline & Panic disorder ( $n$ ) & 0 & 0 & 2 & 0 & 191 & .205 \\
\hline & Agoraphobia (n) & 0 & 0 & 3 & 1 & .321 & .383 \\
\hline & Social phobia (n) & 1 & 1 & 2 & 0 & 321 & .319 \\
\hline & Post-traumatic stress disorder ( $n$ ) & 0 & 0 & 1 & 0 & .438 & .640 \\
\hline & Generalized anxiety disorder (n) & 1 & 1 & 11 & 0 & $.001^{\#}$ & $<.001^{\#}$ \\
\hline & Tic $(0-8)$ & $0.23(0.62)$ & $0.20(0.51)$ & $0.34(0.71)$ & $0.07(0.25)$ & .061 & .088 \\
\hline & BDI (0-39) & $2.13(2.08)$ & $1.59(1.95)$ & $4.62(4.81)$ & $1.64(2.47)$ & $.001 *$ & $.007^{*}$ \\
\hline & STAI $(0-60)$ & $11.68(5.95)$ & $9.02(5.77)$ & $13.95(7.46)$ & $9.41(7.37)$ & $.002 *$ & .340 \\
\hline & STAS $(0-30)$ & $0.23(0.88)$ & $0.00(0.00)$ & $0.43(1.90)$ & $0.12(0.55)$ & .094 & .491 \\
\hline
\end{tabular}

Note: Age: age at the time of MRI examination (in years); Educational attainment (\% low/middle/high): percentage of obsessive-compulsive (OC) symptom high-scoring males, OC symptom low-scoring males, OC symptom high-scoring females, and OC symptom low-scoring females with low, middle, or high educational level. PI-R-ABBR: mean Padua Inventory Abbreviated scores (SD); Y-BOCS severity lifetime: mean Yale-Brown Obsessive-Compulsive Scale severity scores (SD) across whole life span; Y-BOCS symptoms: mean number of symptoms indicated within the aggressive/checking, hoarding/saving symmetry/ordering, washing/cleaning, sexual/religious, and somatic symptom dimensions. MINI (depression, panic disorder, agoraphobia, social disorder, post-traumatic stress disorder, generalized anxiety symmetry/ordering, washing/cleaning, sexual/religious, and somatic symptom dimensions. MINI (depression, panic disorder, agoraphobia, social disorder, post-traumatic stress disorder, generalized anxiety
disorder): number of subjects with current comorbid disorder (measured using the Mini International Neuropsychiatric Interview); Tic: mean tic scores (SD) at the time of MRl; BDI: mean Beck Depression Inventory scores (SD) at the time of MRI; STAl: mean State Trait Anxiety Inventory scores (SD) at the time of MRI; STAS: mean State Trait Anger Scale scores (SD) at the time of MRI.

${ }^{*}$ Significant at $<0.05$ Bonferroni corrected.

"Significant difference in distribution between groups tested using Chi-square, Fisher's exact test. 


\section{Image Acquisition}

The MRI session consisted of an anatomical scan of about 6 minutes. During the scan session, subjects were asked to minimize head movement. To reduce motion artifacts, each participant's head was immobilized using foam pads.

MRI was performed on a 3.0 T Intera MR system (Philips, Medical Systems, Best) with a standard SENSE receiver head coil. The anatomical scan consisted of 182 coronal slices with a 3D T1-weighted gradient-echo sequence (flip angle $8^{\circ}$; repetition time $(\mathrm{TR})=9.69$ milliseconds echo time $(\mathrm{TE})=4.60$ milliseconds matrix, $256 \times 256$ pixels; voxel size, $1.00 \times 1.00 \times 1.20 \mathrm{~mm})$.

\section{Data Analysis}

MRI data were analyzed using SPM8 (Wellcome Department of Imaging Neuroscience, London, UK). T1-weighted MR images were segmented into gray matter, white matter, and cerebrospinal fluid, and normalized to a group template (i.e., a specific template created from the 203 subjects that participated) using the Diffeomorphic Anatomical Registration Through Exponential Lie algebra (DARTEL) algorithm, and subsequently warped from DARTEL space to the standard Montreal Neurological Institute (MNI) brain. To preserve volumetric information, a modulation step was added. Before statistical analysis, the resultant modulated images were spatially smoothed with an $8-\mathrm{mm}$ isotropic Gaussian kernel.

\section{Statistical Tests}

Differences in survey- and interview-based variables were tested using a mixed model analysis of variance (ANOVA; Mixed Models Linear menu item in SPSS) with sex (male vs. female) and OC symptom status (high vs. low) as two fixed factors, and family as a random factor to account for family dependence because the data were collected in twins and siblings. Differences in educational attainment and comorbidity were analyzed using Chi-square statistics (crosstabs; Chi-square, Fisher's exact option in SPSS). Statistical results were considered significant at $p<.05$, Bonferroni corrected.

Differences in regional gray matter volume were tested using the general linear model (full-factorial ANOVA) implemented in SPM8. The design consisted of two independent factors: OC symptom status (high or low scoring) and sex (male or female), and was used to determine the main effect of OC symptom status and the OC symptom status by sex interaction effect. To account for family dependence, brain maps of twin and co-twin of each concordant pair were entered as repeated measures to account for withintwin pair correlations of brain structure. The main effect of OC symptom status was assessed to acquire a general idea of volumetric brain differences between OC symptom high- and low-scoring subjects. The interaction effect of OC symptoms status by sex was assessed by the F-ratio from ANOVA and could be interpreted as OC symptomrelated brain changes that are different in males and females. For significant interactions, we plotted the weighted mean voxel intensities and $90 \%$ confidence intervals (CI) for the most significant coordinate in the region separately for high-scoring and low-scoring males, and high-scoring and low-scoring females to reveal what could explain the observed interaction. Post-hoc tests comparing 'highscoring males with low-scoring males' and 'high-scoring females with low-scoring females' for each coordinate derived from the interaction analysis were considered significant at $p<.01$.

All comparisons were performed with adjustments for total intracranial volume (i.e., the covariate; TIV). Because sex was partially confounded with comorbidity, in particular anxiety and depression (see Table 1), data were reanalyzed with total score on the 13-item Beck Depression Inventory Short Form as an additional covariate. Volumetric changes for the main effect of OC symptoms and the OC symptom by sex interaction effect were assumed significant at $p<.001$ uncorrected with a minimal cluster size of 10 voxels.

\section{Results}

\section{Sample Characteristics}

Sample characteristics are summarized in Table 1. As expected, OC symptom high-scoring subjects (regardless of sex) had significantly higher scores for measurements on OC symptomatology, including OC symptoms as measured with the PI-R-ABBR, as well as OC symptom severity measured with the Yale-Brown Obsessive-Compulsive Scale severity questionnaire. In addition, OC symptom high-scoring subjects were more often diagnosed with current comorbid disorders, mainly anxiety and depression. Furthermore, an interaction between 'OC symptom score' and 'sex' was found for anxiety and depression. This was due to higher levels of comorbid anxiety and depression in high-scoring females compared with high-scoring males.

\section{Main Effect of Obsessive-Compulsive Symptoms}

Differences in gray matter volumes between OC symptom high-scoring subjects and OC symptom low-scoring subjects, regardless of sex, are presented in Table 2 (left side). OC symptom high-scoring subjects had increased gray matter volumes in the right precentral and left middle temporal gyrus and decreased gray matter volumes in the left dorsolateral prefrontal gyrus, left insula, and right substantia nigra. The same results were obtained when depression scores were covaried for (Table 2, right side).

\section{Interaction Effects: OC Symptoms $\times$ Sex}

To examine whether OC symptom related brain changes were different for males and females, the interaction effect 
TABLE 2

Regional Gray Matter Differences in OCS High-Scoring Compared to OCS Low-Scoring Twins

\begin{tabular}{|c|c|c|c|c|c|c|c|c|c|c|c|c|c|c|}
\hline \multicolumn{9}{|c|}{ (A) Covaried for total intracranial volume } & \multicolumn{6}{|c|}{$\begin{array}{l}\text { (B) Covaried for total intracranial volume and } \\
\text { Beck depression inventory scores }\end{array}$} \\
\hline \multirow[b]{2}{*}{ Test } & \multirow[b]{2}{*}{ Anatomical location } & \multirow[b]{2}{*}{ BA } & \multicolumn{3}{|c|}{ MNI coordinates } & \multirow[b]{2}{*}{$T$-value } & \multirow[b]{2}{*}{$p$-value } & \multirow[b]{2}{*}{ \# voxels } & \multicolumn{3}{|c|}{ MNI coordinates } & \multirow[b]{2}{*}{$T$-value } & \multirow[b]{2}{*}{$p$-value } & \multirow[b]{2}{*}{ \# voxels } \\
\hline & & & $x$ & $y$ & $z$ & & & & $x$ & $\mathrm{y}$ & z & & & \\
\hline \multirow[t]{3}{*}{ High > low } & Right precentral & 6 & 66 & -1.5 & 36 & 3.85 & $<.001$ & 75 & 66 & -1.5 & 36 & 3.37 & $<.001$ & 11 \\
\hline & Left middle temporal & 21 & -70.5 & -28.5 & -3 & 3.98 & $<.001$ & 89 & -70.5 & -28.5 & -3 & 3.96 & $<.001$ & 78 \\
\hline & & 21 & -63 & -39 & -4.5 & 3.73 & $<.001$ & 151 & -63 & -39 & -4.5 & 3.57 & $<.001$ & 57 \\
\hline \multirow[t]{3}{*}{ High $<$ low } & Left dorsolateral prefrontal & 9 & -28.5 & 13.5 & 34.5 & 3.46 & $<.001$ & 18 & -28.5 & 13.5 & 34.5 & 3.45 & $<.001$ & 17 \\
\hline & Left insula & 13 & -46.5 & -1.5 & 7.5 & 3.65 & $<.001$ & 73 & -46.5 & 0 & 13.5 & 3.70 & $<.001$ & 67 \\
\hline & Right substantia nigra & & 13.5 & -21 & -13.5 & 3.39 & $<.001$ & 11 & 13.5 & -21 & -12 & 3.44 & $<.001$ & 22 \\
\hline
\end{tabular}

Note: (A) Clusters with regional gray matter differences between obsessive-compulsive (OC) symptom high and low-scoring twins. Test: test for significant gray matter increases (high > low) or decreases (high <low) in OC symptom high relative to OC symptom low-scoring twins; BA: Brodmann area; $\mathrm{MNI}$ coordinates $(\mathrm{mm})$ : location of voxel with the largest effect size; $T$-value: $T$-value of voxel with the largest effect size; p-value: cluster $p$-value; \# voxels: number of voxels in cluster.

(B) Clusters with regional gray matter differences between OC symptom high and low-scoring twins after adjusting for the Beck-Depression Inventory scores.

TABLE 3

Interaction effect of OCS $\times$ Sex in Regional Gray Matter

\begin{tabular}{|c|c|c|c|c|c|c|c|c|c|c|c|c|c|}
\hline \multicolumn{8}{|c|}{ (A) Covaried for total intracranial volume } & \multicolumn{6}{|c|}{$\begin{array}{l}\text { (B) Covaried for total intracranial volume and Beck } \\
\text { depression inventory scores }\end{array}$} \\
\hline \multirow[b]{2}{*}{ Anatomical location } & \multirow[b]{2}{*}{ BA } & \multicolumn{3}{|c|}{ MNI coordinates } & \multirow[b]{2}{*}{ F-value } & \multirow[b]{2}{*}{$p$-value } & \multirow[b]{2}{*}{ \# voxels } & \multicolumn{3}{|c|}{$\mathrm{MNI}$ coordinates } & \multirow[b]{2}{*}{ F-value } & \multirow[b]{2}{*}{$p$-value } & \multirow[b]{2}{*}{ \# voxels } \\
\hline & & $x$ & $y$ & z & & & & $x$ & $y$ & z & & & \\
\hline \multicolumn{14}{|c|}{$\begin{array}{l}\text { Larger gray matter volumes in males with high OC symptomatology versus males with low OC symptomatology, with no effect or a reversed } \\
\text { effect of OC symptomatology in females }\end{array}$} \\
\hline \multirow[t]{2}{*}{ Left middle temporal } & 21 & -70.5 & -30 & -3 & 12.50 & $<.001$ & 12 & & & & & & \\
\hline & 21 & -70.5 & -43.5 & -7.5 & 14.40 & $<.001$ & 33 & -70.5 & -43.5 & -7.5 & 16.15 & $<.001$ & 57 \\
\hline Right middle temporal & 39 & 45 & -58.5 & 3 & 16.57 & $<.001$ & 104 & 45 & -58.5 & 3 & 18.52 & $<.001$ & 153 \\
\hline \multicolumn{14}{|c|}{$\begin{array}{l}\text { Larger gray matter volumes in females with high OC symptomatology versus females with low OC symptomatology with no effect or a reversed } \\
\text { effect of OC symptomatology in males }\end{array}$} \\
\hline Right precuneus & 7 & 18 & -37.5 & 54 & 17.53 & $<.001$ & 227 & 18 & -37.5 & 54 & 17.73 & $<.001$ & 249 \\
\hline
\end{tabular}

of OC symptoms by sex was investigated. To reveal what could explain these interactions, weighted mean intensities (contrast estimates) and $90 \%$ confidence intervals were plotted for each significant coordinate derived from the interaction analysis (Figure 1). Post-hoc tests indicated that our finding of a regional enlargement in the left middle temporal gyrus in subjects scoring high for OC symptoms was completely driven by a larger gray matter volume for this region in OC symptom high-scoring males (Table 3, left side). In addition, a region within the right middle temporal gyrus was found to be larger in OC symptom high-scoring males, but reduced in OC symptom high-scoring females. This opposite finding in males and females acted to hide the OC symptom main effect in the right middle temporal gyrus. A region within the right precuneus was found to be larger in OC symptom high-scoring females, but reduced in OC symptom high-scoring males. This region was again not found in the OC symptom main effect. Same results were obtained when depression scores were covaried for (Table 3, right side).

\section{Discussion}

This study investigated whether sex could be a potential source of heterogeneity in brain imaging outcomes on OC symptomatology. To assess how OC symptomatology affects the brain we first compared gray matter volumes from twins (and siblings) with high OC symptom scores with those from twins with low OC symptom scores, regardless of sex. Regions that were found to be larger in twins with high OC symptom scores included the right precentral and the left middle temporal gyrus, whereas the left dorsolateral prefrontal gyrus, the left insula, and the right substantia nigra were found to be reduced in OC symptom high-scoring twins. Our finding of a reduced dorsolateral prefrontal gyrus is consistent with previous studies (see meta-analysis 

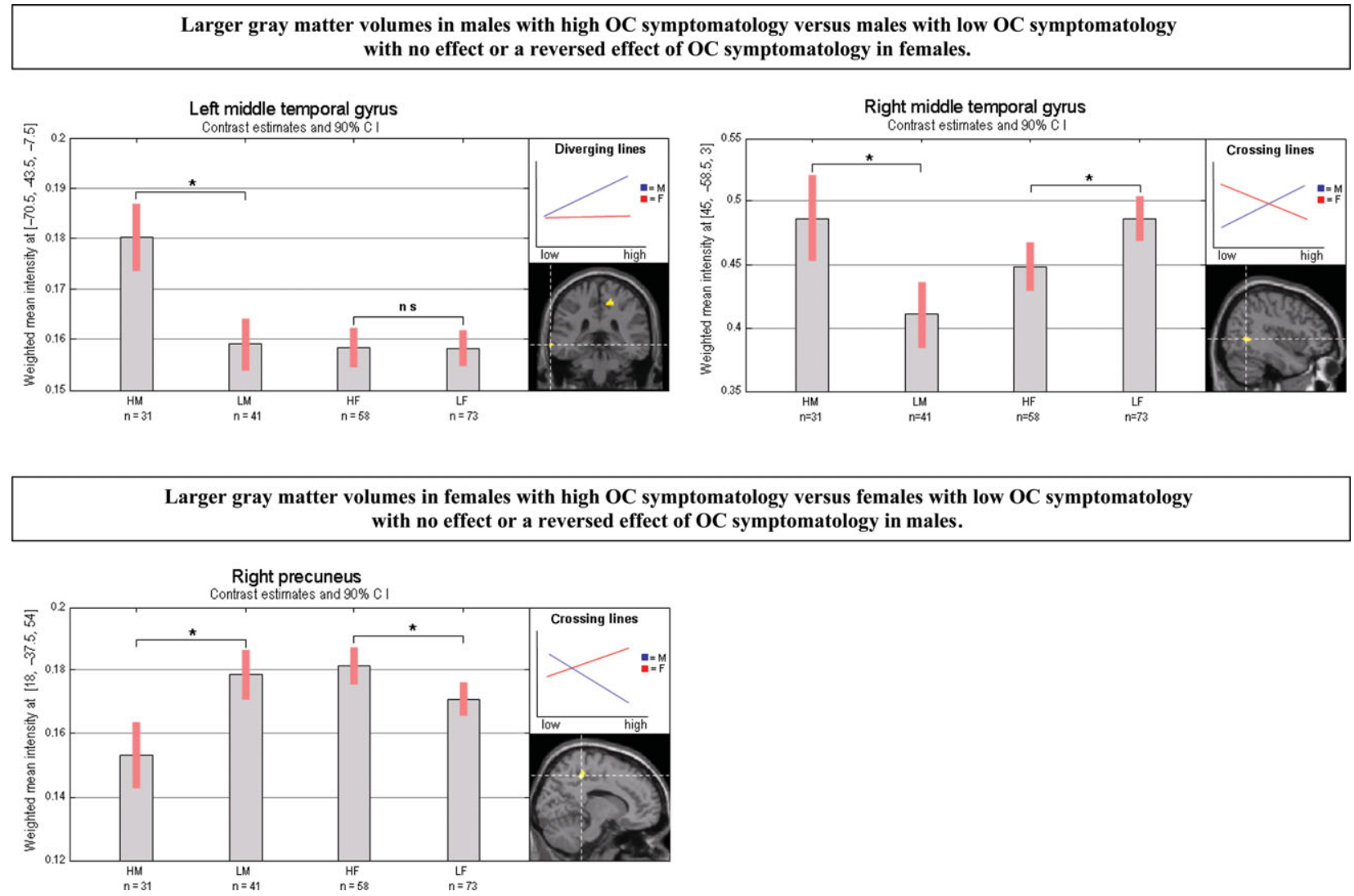

\section{FIGURE 1}

Obsessive-compulsive $(\mathrm{OC})$ symptom status-related brain changes that are different in males and females. Bar graphs indicate the weighted mean intensities (contrast estimates) and $90 \%$ confidence intervals $(\mathrm{Cl})$ for each coordinate derived from the OC symptom status by sex interaction test separately for the OC symptom high-scoring males (HM), OC symptom low-scoring males (LM), OC symptom high-scoring females (HF), and OC symptom low-scoring females (LF). Note: *Post-hoc tests ('high-scoring males versus low-scoring males' or 'high-scoring females versus low-scoring females') significant at $p<.01$; ns = post-hoc tests not significant. Diverging lines can act to reduce or hide the main effect. Crossing lines are likely to hide the main effect.

by Radua \& Mataix-Cols, 2009). Together with the substantia nigra, this region has been implicated in the dorsolateral prefrontal-striatal loop of the CSTC network (Cummings, 1993) that has been associated with OC symptomatology (Mataix-Cols \& van den Heuvel, 2006). A reduced insular volume also replicates previous findings (Pujol et al., 2004; Soriano-Mas et al., 2007) and this structure has been mainly linked to OC symptomatology through its involvement in the neurocircuitry of disgust (Husted et al., 2006).

The main focus of this article was whether OC symptomrelated brain changes are different for males and females and whether this difference may have acted to hide or reduce a main effect in our study, or in the mixed sex samples studied previously. In order to investigate this, the interaction effect of OC symptoms by sex on gray matter volume was assessed. This OC symptom by sex interaction analysis indicates that our finding of increased left middle temporal volume in OC symptom high-scoring twins was completely driven by a larger gray matter volume for this region in OC symptom high-scoring males. The OC symptom by sex interaction analysis also revealed opposite effects of OC symptomatology in males and females for the right middle temporal gyrus and the right precuneus. OC symptom high-scoring males were found to have a larger right middle temporal gyrus, whereas in high-scoring females this region was reduced. Conversely, the right precuneus was found to be larger in OC symptom high-scoring females, but reduced in OC symptom high-scoring males. These opposite findings in males and females acted to hide the OC symptom main effect. The middle temporal gyrus is involved in verbal memory and auditory processing (Binder et al., 1994; Boly et al., 2004; Grasby et al., 1993), and precuneus function has been associated with higher order cognitive processes, including visuo-spatial processing, episodic memory retrieval, and planning (Cavanna \& Trimble, 2006). Both regions have been implicated in the neuroanatomical model for OCD predominantly through their functional connections with the dorsal and ventral prefrontal cortex. However, results from structural and functional imaging studies have provided inconsistent results regarding the direction of 
anatomical and functional changes for these specific brain regions (Menzies et al., 2008).

Based on our results, we hypothesize that the use of mixed sex samples with unequal distribution of males and females between studies (e.g., more males in one study vs. more females in another study) may have contributed to these opposite findings. With regard to middle temporal and precuneus volumes, a review of the current literature supports our hypothesis directly. For example, Kim and colleagues (2001) observed increased gray matter in the right temporal gyrus in a sample that included a higher number of males compared with females (patients: 17 males vs. 8 females), whereas Togao and colleagues (2010) observed decreased gray matter for this region in a sample that included a higher number of females compared with males (patients: 9 males vs. 14 females). In addition, with respect to the right precuneus, Pujol and colleagues (2004) found a tendency toward decreased gray matter for this region in a sample that included more males than females (patients: 40 males vs. 32 females). This finding was replicated and found to be significant in a patient sample comprising 21 males and 9 females (Soriano-Mas et al., 2007). This latter study also assessed the feasibility of classifying single subject cases of MRI data as OCD patients or healthy controls using their whole brain anatomy, and found that including gender in their analyses improved their classification accuracy (Soriano-Mas et al., 2007), which further supports our finding that OC symptom-related brain changes can be different in males and females.

Previous studies have shown that OC symptomatology and its developmental trajectories tend to be distinct in males and females, where females tend to report more contamination obsessions and cleaning compulsions (Castle et al., 1995; Labad et al., 2008; Lensi et al., 1996; Noshirvani et al., 1991; Tukel et al., 2004), whereas symmetry, religious and sexual obsessions (Labad et al., 2008; Lensi et al., 1996; Tukel et al., 2004), and an earlier onset of the disorder is more common in males (Bogetto et al., 1999; Castle et al., 1995; Labad et al., 2008; Noshirvani et al., 1991; Tukel et al., 2004). Interestingly, distinct neural correlates for these specific OC symptom dimensions have been found in both brain structure as well as brain function during specific symptom provocation (MataixCols et al., 2004; van den Heuvel et al., 2009). The 'male' symmetry/ordering dimension was found to be negatively correlated with regional gray matter volume in the motor cortex, insula, and parietal cortex (which includes the precuneus) and positively correlated with temporal gray and white matter volume. The finding of a smaller precuneus and a larger middle temporal lobe in the males with high OC symptomatology, with opposite or no effects in females, suggests that the brain regions differentially affected in males and females may be intimately connected to the difference in patterns of OC symptomatology between sexes.
In summary, this study shows that OC symptom-related changes in the left middle temporal gyrus, right middle temporal gyrus, and right precuneus are different for males and females. These findings might, in part, explain inconsistencies in the previous literature and show the importance of taking sex into account when investigating the neurobiology of OC symptoms.

\section{Acknowledgments}

We thank Gabriëlla Blokland, Myrle Kemperman, Judith Wagner, Mira Geirnaert, Kim Meijer and Daisy van Minde for help with MRI data. This work was supported by the Netherlands Organisation for Scientific Research [MW 90461-193, MaGW-nr: 400-07-080, MagW 480-04-004] and the Neuroscience Campus Amsterdam [AC-2009-F2-3.]

\section{References}

American Psychiatric Association. (1994). Diagnostic and statistical manual of mental disorder: DSM-IV (4th ed.). Washington, DC: American Psychiatric Association.

Beck, A. T., Rial, W. Y., \& Rickels, K. (1974). Short form of depression inventory: Cross-validation. Psychological Reports, 34, 1184-1186.

Beck, A. T., Ward, C. H., Mendelson, M., Mock, J., \& Erbaugh, J. (1961). An inventory for measuring depression. Archives of General Psychiatry, 4, 561-571.

Binder, J. R., Rao, S. M., Hammeke, T. A., Yetkin, F. Z., Jesmanowicz, A., Bandettini, P. A., ... Haughton, V. M. (1994). Functional magnetic resonance imaging of human auditory cortex. Annals of Neurology, 35, 662-672.

Bogetto, F., Venturello, S., Albert, U., Maina, G., \& Ravizza, L. (1999). Gender-related clinical differences in obsessive-compulsive disorder. European Psychiatry, 14, 434-441.

Boly, M., Faymonville, M. E., Peigneux, P., Lambermont, B., Damas, P., Del, F. G., . . Laureys, S. (2004). Auditory processing in severely brain injured patients: Differences between the minimally conscious state and the persistent vegetative state. Archives of Neurology, 61, 233-238.

Castle, D. J., Deale, A., \& Marks, I. M. (1995). Gender differences in obsessive-compulsive disorder. Australian and New Zealand Journal of Psychiatry, 29, 114-117.

Cath, D. C., van Grootheest, D. S., Willemsen, G., van Oppen, P., \& Boomsma, D. I. (2008). Environmental factors in obsessive-compulsive behavior: Evidence from discordant and concordant monozygotic twins. Behavior Genetics, 38, 108-120.

Cavanna, A. E., \& Trimble, M. R. (2006). The precuneus: A review of its functional anatomy and behavioural correlates. Brain, 129, 564-583.

Cosgrove, K. P., Mazure, C. M., \& Staley, J. K. (2007). Evolving knowledge of sex differences in brain structure, function, and chemistry. Biological Psychiatry, 62, 847-855.

Cummings, J. L. (1993). Frontal-subcortical circuits and human behavior. Archives of Neurology, 50, 873-880. 
den Braber, A., van't Ent, D., Boomsma, D. I., Cath, D. C., Veltman, D. J., Thompson, P. M., \& de Geus, E. J. (2011). White matter differences in monozygotic twins discordant or concordant for obsessive-compulsive symptoms: A combined diffusion tensor imaging/voxel-based morphometry study. Biological Psychiatry, 70, 969-977.

den Braber, A., van 't Ent, D., Cath, D. C., Wagner, J., Boomsma, D. I., \& de Geus, E. J. (2010). Brain activation during cognitive planning in twins discordant or concordant for obsessive-compulsive symptoms. Brain, 133, 31233140.

Friedlander, L., \& Desrocher, M. (2006). Neuroimaging studies of obsessive-compulsive disorder in adults and children. Clinical Psychology Review, 26, 32-49.

Fullana, M. A., Mataix-Cols, D., Caspi, A., Harrington, H., Grisham, J. R., Moffitt, T. E., \& Poulton, R. (2009). Obsessions and compulsions in the community: Prevalence, interference, help-seeking, developmental stability, and cooccurring psychiatric conditions. American Journal of Psychiatry, 166, 329-336.

Goodman, W. K., Price, L. H., Rasmussen, S. A., Mazure, C., Delgado, P., Heninger, G. R., \& Charney, D. S. (1989a). The Yale-Brown obsessive compulsive scale. II. Validity. Archives of General Psychiatry, 46, 1012-1016.

Goodman, W. K., Price, L. H., Rasmussen, S. A., Mazure, C., Fleischmann, R. L., Hill, C. L., ... Charney, D. S. (1989b). The Yale-Brown obsessive compulsive scale I. Development, use, and reliability. Archives of General Psychiatry, 46, 10061011.

Grabe, H. J., Meyer, C., Hapke, U., Rumpf, H. J., Freyberger, H. J., Dilling, H., \& John, U. (2000). Prevalence, quality of life and psychosocial function in obsessive-compulsive disorder and subclinical obsessive-compulsive disorder in northern Germany. European Archives of Psychiatry and Clinical Neuroscience, 250, 262-268.

Grasby, P. M., Frith, C. D., Friston, K. J., Bench, C., Frackowiak, R. S., \& Dolan, R. J. (1993). Functional mapping of brain areas implicated in auditory — verbal memory function. Brain, 116 (Pt 1), 1-20.

Husted, D. S., Shapira, N. A., \& Goodman, W. K. (2006). The neurocircuitry of obsessive-compulsive disorder and disgust. Progress in Neuro-Psychopharmacology \& Biological Psychiatry, 30, 389-399.

Kim, J. J., Lee, M. C., Kim, J., Kim, I. Y., Kim, S. I., ... Kwon, J. S. (2001). Grey matter abnormalities in obsessivecompulsive disorder: Statistical parametric mapping of segmented magnetic resonance images. British Journal of Psychiatry, 179, 330-334.

Labad, J., Menchon, J. M., Alonso, P., Segalas, C., Jimenez, S., Jaurrieta, N., ... Vallejo, J. (2008). Gender differences in obsessive-compulsive symptom dimensions. Depression and Anxiety, 25, 832-838.

Lenroot, R. K., \& Giedd, J. N. (2010). Sex differences in the adolescent brain. Brain and Cognition, 72, 46-55.

Lensi, P., Cassano, G. B., Correddu, G., Ravagli, S., Kunovac, J. L., \& Akiskal, H. S. (1996). Obsessive-compulsive disorder. Familial-developmental history, symptomatology, comorbidity and course with special reference to gender- related differences. British Journal of Psychiatry, 169, 101107.

Mataix-Cols, D., \& van den Heuvel, O. A. (2006). Common and distinct neural correlates of obsessive-compulsive and related disorders. Psychiatric Clinics of North America, 29, 391-410, viii.

Mataix-Cols, D., Wooderson, S., Lawrence, N., Brammer, M. J., Speckens, A., \& Phillips, M. L. (2004). Distinct neural correlates of washing, checking, and hoarding symptom dimensions in obsessive-compulsive disorder. Archives of General Psychiatry, 61, 564-576.

McCarthy, M. M., \& Arnold, A. P. (2011). Reframing sexual differentiation of the brain. Nature Neuroscience, 14, 677683.

Menzies, L., Chamberlain, S. R., Laird, A. R., Thelen, S. M., Sahakian, B. J., \& Bullmore, E. T. (2008). Integrating evidence from neuroimaging and neuropsychological studies of obsessive-compulsive disorder: The orbitofronto-striatal model revisited. Neuroscience \& Biobehavioral Reviews, 32, 525-549.

Noshirvani, H. F., Kasvikis, Y., Marks, I. M., Tsakiris, F., \& Monteiro, W. O. (1991). Gender-divergent aetiological factors in obsessive-compulsive disorder. British Journal of Psychiatry, 158, 260-263.

Pujol, J., Soriano-Mas, C., Alonso, P., Cardoner, N., Menchon, J. M., Deus, J., \& Vallejo, J. (2004). Mapping structural brain alterations in obsessive-compulsive disorder. Archives of General Psychiatry, 61, 720-730.

Rachman, S., \& de Silva, P. (1978). Abnormal and normal obsessions. Behaviour Research and Therapy, 16, 233248 .

Radua, J., \& Mataix-Cols, D. (2009). Voxel-wise meta-analysis of grey matter changes in obsessive-compulsive disorder. British Journal of Psychiatry, 195, 393-402.

Radua, J., van den Heuvel, O. A., Surguladze, S., \& Mataix-Cols, D. (2010). Meta-analytical comparison of voxel-based morphometry studies in obsessive-compulsive disorder vs. other anxiety disorders. Archives of General Psychiatry, 67, 701-711.

Rotge, J. Y., Guehl, D., Dilharreguy, B., Tignol, J., Bioulac, B., Allard, M., ... Aouizerate, B. (2009). Meta-analysis of brain volume changes in obsessive-compulsive disorder. Biological Psychiatry, 65, 75-83.

Salkovskis, P. M., \& Harrison, J. (1984). Abnormal and normal obsessions - A replication. Behaviour Research and Therapy, 22, 549-552.

Saxena, S., \& Rauch, S. L. (2000). Functional neuroimaging and the neuroanatomy of obsessive-compulsive disorder. Psychiatric Clinics of North America, 23, 563-586.

Sheehan, D. V., Lecrubier, Y., Sheehan, K. H., Amorim, P., Janavs, J., Weiller, E., .. . Dunbar, G. C. (1998). The MiniInternational Neuropsychiatric Interview (MINI): The development and validation of a structured diagnostic psychiatric interview for DSM-IV and ICD-10. Journal of Clinical Psychiatry, 59(Suppl. 20), 22-33.

Sisk, C. L., \& Zehr, J. L. (2005). Pubertal hormones organize the adolescent brain and behavior. Front Neuroendocrinol, 26, 163-174. 
Soriano-Mas, C., Pujol, J., Alonso, P., Cardoner, N., Menchon, J. M., Harrison, B. J., ... Gaser, C. (2007). Identifying patients with obsessive-compulsive disorder using whole-brain anatomy. Neuroimage, 35, 10281037.

Spielberger, C. D., Gorsuch, R. L., \& Lusehe, R. E. (1970). Manual for the state-trait anxiety inventory (STAI). Palo Alto, CA: Consulting Psychologists Press.

Spielberger, C. D., Jacobs, G. A., Russell, S., \& Crane, R. S. (1983). Assessment of anger: The state trait anger scale (Vol. 2). Hillsdale, NJ: Lawrence Erlbaum.

Togao, O., Yoshiura, T., Nakao, T., Nabeyama, M., Sanematsu, H., Nakagawa, A., ... Honda, H. (2010). Regional gray and white matter volume abnormalities in obsessive- compulsive disorder: A voxel-based morphometry study. Journal of Psychiatric Research, 184, 29-37.

Tukel, R., Polat, A., Genc, A., Bozkurt, O., \& Atli, H. (2004). Gender-related differences among Turkish patients with obsessive-compulsive disorder. Comprehensive Psychiatry, 45, 362-366.

van den Heuvel, O. A., Remijnse, P. L., Mataix-Cols, D., Vrenken, H., Groenewegen, H. J., Uylings, H. B., ... Veltman, D. J. (2009). The major symptom dimensions of obsessive-compulsive disorder are mediated by partially distinct neural systems. Brain, 132, 853-868.

van Oppen, P., Hoekstra, R. J., \& Emmelkamp, P. M. (1995). The structure of obsessive-compulsive symptoms. Behaviour Research and Therapy, 33, 15-23. 